\title{
ÁREAS DE INFLUENCIA Y COMPETENCIA ESPACIAL DE GRANDES SUPERFICIES COMERCIALES: UNA APROXIMACIÓN EN EL CASO DE ZARAGOZA
}

\section{Carmen Díez Cornago (1) y Ana Isabel Escalona Orcao (2)}

(1) Instituto Nacional de Meteorología, Zaragoza

(2) Dpto. de Geografía y Ordenación del Territorio, Universidad de Zaragoza

\begin{abstract}
Resumen: El artículo plantea un estudio sobre las áreas de influencia y la competitividad espacial que reflejan, en el caso de tres grandes superficies comerciales de la ciudad de Zaragoza. Para ello se combinan las ventajas de la modelización gravitatoria con las de las encuestas a los compradores, con objeto de establecer cuotas de mercado expresivas de la penetración de los centros estudiados en los distintos distritos urbanos. Se contribuye así no sólo a mejorar el conocimiento de la geografía comercial zaragozana, sino también a confirmar la validez exploratoria de métodos como los citados, especialmente de cara al planteamiento de hipótesis que puedan sustentar ulteriores investigaciones.
\end{abstract}

Palabras clave: áreas de influencia, competitividad, grandes superficies, métodos gravitatorios.

Abstract: The aim of the article is the determination of market areas and spatial competition in the case of three shopping centres in the urban areas of Zaragoza. The research was conducted in several stages, including the application of a gravitational model and the survey to consumers, in order to establish market shares and to calculate the market penetration of each shopping center in each urban district or area. The results support the usefulness of the model in describing the market share of shopping centres with reasonable accuracy, and bring a better knowledge of the commercial geography in Zaragoza as well.

Key words: market areas, spatial competition, shopping centres, gravitational models. 


\section{Introducción ${ }^{1}$}

La presencia de grandes superficies comerciales en los espacios urbanos sigue suscitando interés y más en el caso de aquellos espacios — como el de la ciudad de Zaragoza - donde su implantación ha sido más reciente. Son muchos los aspectos que han atraído a los investigadores españoles, desde el seguimiento de su progresiva aparición y la identificación de las principales empresas implicadas, pasando por la estimación de su impacto sobre la estructura comercial preexistente o sobre su entorno urbano inmediato. De los trabajos resultantes pueden dar idea diversas publicaciones colectivas, algunas de las cuales constituyen las actas de sucesivas reuniones científicas celebradas durante el último decenio. Es el caso de "Primeras Jornadas de Geografía y Comercio: Grandes superficies comerciales", celebradas en 1990 (VV.AA., 1991); o de "La geografía de los servicios en España" (Antón Burgos, 1999).

Con respecto al carácter de la mayor parte de tales trabajos podrá seguramente hacérseles extensiva la afirmación de Escolano (1999), en el sentido de que entre los estudios geográficos sobre el comercio escasean los de carácter teórico y proliferan, sin embargo, los de carácter más analítico. Ciertamente no debe ello extrañar, si se tiene en cuenta el notable desarrollo de métodos y técnicas susceptibles de ser aplicados en este campo, así como su atractivo; circunstancias ambas que concurren especialmente en relación con las grandes superficies comerciales (Moreno, 1995)

En el caso de este artículo, su justificación sería precisamente la ya mencionada, a saber, aprovechar el potencial analítico del modelo de Huff, en la versión sugerida por Berry y Parr (1988), y aplicarlo al estudio de las áreas de influencia comercial de tres grandes superficies que estaban en servicio en la ciudad de Zaragoza cuando el trabajo se inicia (noviembre de 1999). Pretendemos también realizar una aportación al conocimiento de la geografía comercial zaragozana, donde se cuenta con trabajos valiosos aunque no muy abundantes (Bielza y Escolano, 1985; Escolano, 1985; Escolano, 1995; Báguena, 1998). Para ello, tras el indispensable preámbulo conceptual-metodológico y la presentación del espacio de estudio, el trabajo se centra en la comparación de dos técnicas distintas para la delimitación de las áreas comerciales: los modelos gravitatorios convencionales y los que incorporan elementos de la realidad a partir de encuestas. Con los datos obtenidos, probabilidades teóricas primero y cuotas de mercado después, se ha realizado la correspondiente cartografía, expresiva -aunque con diferencias- del alcance espacial de las áreas de mercado así como de la penetración de los centros comerciales en los distintos distritos de la ciudad.

Los resultados obtenidos han confirmado la validez exploratoria del método, así como su idoneidad de cara a plantear investigaciones más ambiciosas. Pero también se ha evidenciado, una vez más, la buena salud de formulaciones ya clásicas en el acervo de los geógrafos, y el interés con el que las reciben las nuevas generaciones, 
tal y como tuvimos ocasión de comprobar con los alumnos de la segunda promoción del plan nuevo del Departamento de Geografía de Zaragoza, en cuyas clases prácticas se gestó este trabajo.

\section{Consideraciones de carácter conceptual y metodológico}

Con carácter general podríamos situar esta investigación en el amplio campo que brinda el marketing al trabajo de los geógrafos. Las obras y autores de referencia han sido recordados por Moreno (1995), quien señala además los tres elementos o líneas de trabajo básicos en el campo del llamado marketing empresarial, a saber: el estudio de la segmentación del mercado, el análisis de la idoneidad de los posibles emplazamientos, y la delimitación del área de mercado o de ventas (Moreno, 1995: 123). La segunda y la tercera de ambas temáticas confirman la vigencia del tradicional concepto de "área de influencia", que efectivamente ha sido utilizado para valorar diversas cualidades del emplazamiento de una gran superficie, como su accesibilidad, su volumen de demanda potencial, las expectativas de gasto suscitadas por los distintos segmentos de hogares o, en definitiva, su idoneidad para optimizar el rendimiento de la empresa. Pero cuando se trata de servicios a la población, como en este caso, también la propia dimensión del área de influencia, o área de mercado, resulta expresiva por sí misma, ya que — dada la exacerbada competitividad interempresarial--, constituye un elemento valioso para apreciar la posición competitiva de cualquier establecimiento comercial en el espacio urbano.

El presente trabajo asume precisamente esa última acepción del concepto de área de influencia para estudiar cómo se reparten el espacio urbano de Zaragoza tres grandes superficies comerciales. Desde el punto de vista metodológico son numerosas las formas de delimitación de áreas de mercado, y además están plenamente consolidadas: es el caso del trazado de isocronas y de las encuestas a los consumidores, pasando por la modelización gravitatoria. De su utilización, tanto separada como conjuntamente con otros métodos más complejos e incluso mediante SIG, hay ejemplos en la bibliografía española (Serrano y Martín, 1990; Delgado, 1990; Escolano, 1995; Moreno y colbs., 1999). No obstante — sobre todo en etapas exploratorias-, se les sigue reconociendo a los modelos gravitatorios de tipo probabilista, como el de Huff, una notable utilidad para delimitar y predecir las áreas de mercado con precisión. Pero también es cierto que sus resultados mejoran si se enriquece el análisis con diversos parámetros específicos, obtenidos algunos a partir de encuestas, que den más verosimilitud a las resultantes cuotas de mercado de cada centro comercial en cada área de estudio. Es lo que hacen entre otros Berry y Pahr (1988), cuya metodología hemos seguido de cerca en el caso de Zaragoza. 
Tomando como ejemplo el centro comercial Grancasa, se comprobará cómo los resultados cartográficos según los dos métodos son distintos, conforme a los supuestos asumidos en cada caso: omisión de cualquier otra posible oferta y restricción de la impedancia espacial $\longrightarrow$ dificultad de acceso desde cada centroide - a una función únicamente del tiempo de desplazamiento, en la aplicación sencilla del modelo de Huff; y en el estudio mediante cuotas de mercado, presunción de competencia de otras ofertas locales del mismo rango y extensión de la impedancia espacial -entendida en esta ocasión como función no sólo de tiempo de desplazamiento sino de otros atributos de la red como su coste, conveniencia, fiabilidad, seguridad, etc. Tendremos pués, con ambos mapas, dos ejemplos claros de esa "geometría variable" (Escolano, 1999: 448) característica de la organización espacial de la actividad comercial, así como una justificación más para mejorar nuestro conocimiento sobre cuáles son realmente los factores que determinan el atractivo, la frecuentación real y, en definitiva, la competitividad espacial de las grandes superficies comerciales.

\section{Presentación del espacio comercial zaragozano y de los elementos del estudio}

El espacio de referencia es el término municipal de Zaragoza, cuya población en 1999 era de 608.180 habitantes en una extensión de $1.063,10 \mathrm{~km}^{2}$. Por el carácter experimental del análisis no se ha contemplado la posibilidad de que la atracción de las grandes superficies comerciales rebase, como así es, tal espacio. En cuanto a los establecimientos estudiados, han sido los tres más importantes abiertos en Zaragoza a la fecha del estudio, noviembre de 1999, es decir: el hipermercado Alcampo Utebo, situado en el colindante municipio del mismo nombre y cuyo término municipal ha sido igualmente incluido en el estudio; el centro comercial Augusta y el centro comercial Grancasa. A este último pertenecen los datos y mapas con los que se describe la metodología, entre otras cosas por ser el más reciente hasta la fecha del estudio (se abrió en 1997) y el mayor de todos ( $78.188 \mathrm{~m}^{2}$ de superficie bruta alquilable). Las fechas de apertura de los centros Alcampo y Augusta habían sido 1981 y 1995 respectivamente, y sus superficies brutas alquilables ascendian a 21.244 y $50.800 \mathrm{~m}^{2}$ también respectivamente. Pese a las inevitables diferencias todos ellos encajan en la definición para tales establecimientos realizada por la Asociación Española de Centros Comerciales (Flavián y Polo, 1997)2.

Como se ve en el mapa de situación (figura 1), el hipermercado Alcampo se encuentra a $12 \mathrm{~km}$ de la ciudad en dirección NW sobre la carretera de Logroño, y pertenece ya al vecino término municipal de Utebo (razón por la cual se le ha incluido también en el estudio). En el mismo eje pero lindando con el propio espacio urbano se encuentra el centro comercial Augusta, mientras que el centro comer- 


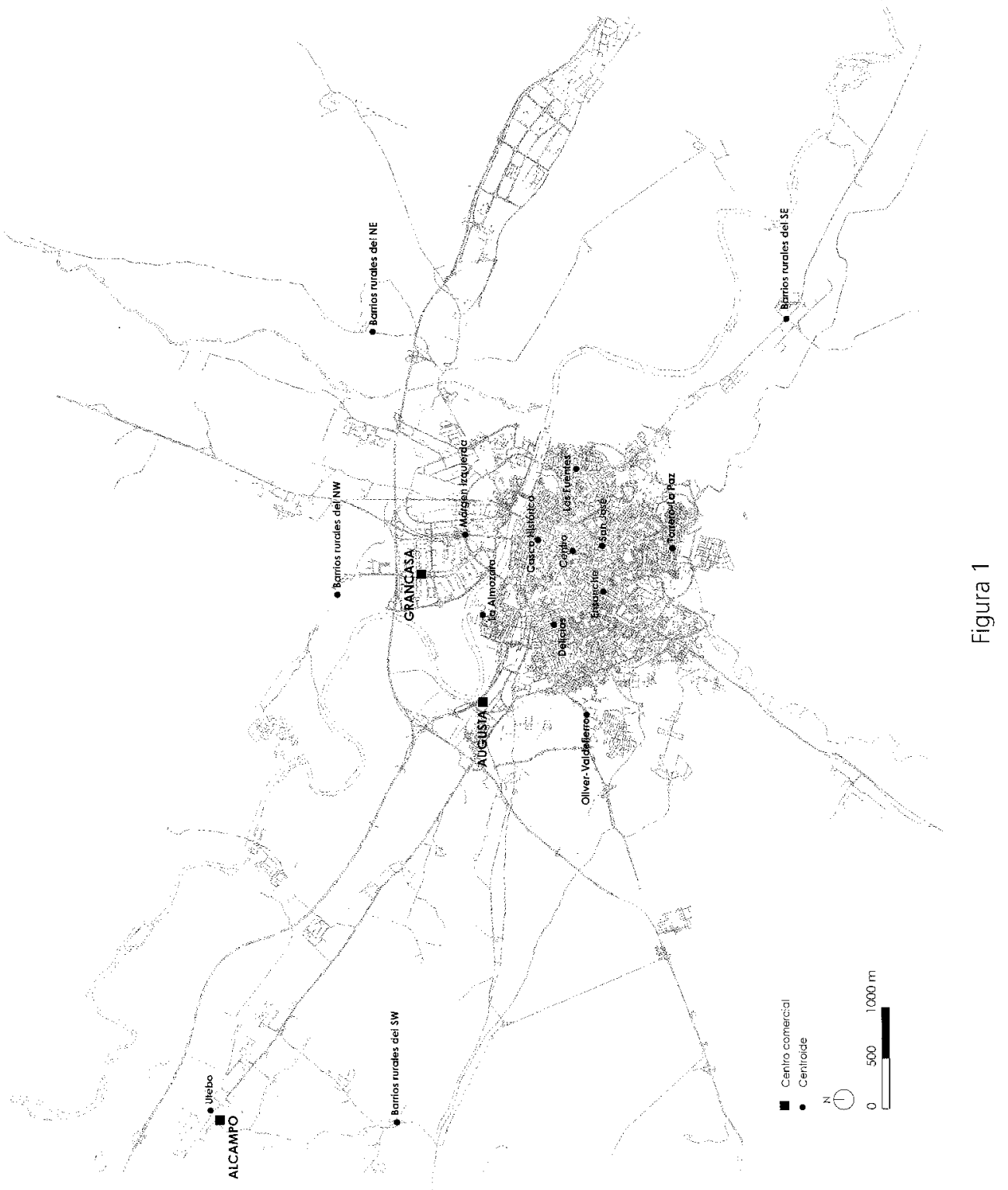


cial Grancasa se ubica en la zona de expansión urbana surgida en la margen izquierda del río Ebro al norte de la ciudad. Si los tres centros constituyen destinos en competencia de los compradores zaragozanos, los orígenes se han situado en los centroides de quince áreas que hemos llamado distritos, ya que catorce de ellas lo son del término municipal de Zaragoza, y la decimoquinta corresponde al término de Utebo.

Para quien no conozca Zaragoza puede ser útil observar además en las tablas 1 y 2 algunas características interesantes de dichos distritos, como las diferencias en su población y dotación comercial respectiva, (según el ejemplo del comercio minorista). Se aprecia la notable masificación poblacional ya que 5 distritos (Centro, Delicias, Ensanche, San José y Las Fuentes) suman en 1996 el 58,1\% de la población; hecho que se corresponde con una también grande, aunque menos marcada, concentración de la oferta comercial, donde destaca además el casco antiguo. Y puede comprobarse la correlación que suele darse entre la evolución de ambas variables, con retroceso de la oferta comercial en los distritos en descenso demográfico, y por el contrario - aumento de la misma en las áreas demográficamente expansivas. Es claramente el caso del Distrito de Margen Izquierda, donde se abre el centro comercial Grancasa, y al que nos referiremos después en el análisis.

Tabla 1. Población de derecho por distritos censales

\begin{tabular}{|l|c|c|c|c|c|}
\cline { 2 - 6 } \multicolumn{1}{c|}{} & 1991 & $\begin{array}{c}\text { \% sobre total } \\
1991\end{array}$ & 1996 & $\begin{array}{c}\text { \% sobre total } \\
1996\end{array}$ & $\begin{array}{c}\text { Tasa variación } \\
1996 / 1991\end{array}$ \\
\hline 1.- - Casco Histórico & 41.025 & 6,9 & 38.907 & 6,5 & $-5,16$ \\
\hline 2.- Centro & 63.721 & 10,7 & 60.489 & 10,1 & $-5,07$ \\
\hline 3.- Delicias & 112.173 & 18,9 & 111.915 & 18,6 & $-0,23$ \\
\hline 4.- Ensanche & 60.692 & 10,2 & 59.234 & 9,8 & $-2,40$ \\
\hline 5.- San José & 72.488 & 12,2 & 69.216 & 11,5 & $-4,51$ \\
\hline 6.- Las Fuentes & 49.390 & 8,3 & 48.485 & 8,1 & $-1,83$ \\
\hline 7.- Almozara & 29.284 & 4,9 & 26.591 & 4,4 & $-9,19$ \\
\hline 8.- Oliver - Valdefierro & 23.425 & 3,9 & 25.038 & 4,2 & 6,89 \\
\hline 9.- Torrero - La Paz & 34.616 & 5,8 & 34.665 & 5,8 & 0,14 \\
\hline 10.- Margen Izquierda & 81.376 & 13,7 & 96.669 & 16,1 & 18,79 \\
\hline 11.- - Barrios Rurales Norte & 15.209 & 2,6 & 17.986 & 3,0 & 18,26 \\
\hline 12.- Barrios Rurales Sur & 10.995 & 1,8 & 12.479 & 2,1 & 13,50 \\
\hline
\end{tabular}

Fuente: Padrón Municipal de Habitantes, año 1996. Ayuntamiento de Zaragoza Instituto Aragonés de Estadistica. Censo de la Población 1991, INE 
Tabla 2. Cambios en la distibución y localización del comercio

\begin{tabular}{|l|c|c|c|}
\cline { 2 - 4 } & $\begin{array}{c}\text { \% sobre total } \\
1991\end{array}$ & $\begin{array}{c}\% \text { sobre total } \\
1996\end{array}$ & $\begin{array}{c}\text { Tasa variación } \\
1996 / 1991\end{array}$ \\
\hline 1.- Casco Histórico & 13,78 & 12,31 & $-8,57$ \\
\hline 2.- Centro & 18,12 & 18,68 & 5,58 \\
\hline 3.- Delicias & 20,33 & 21,53 & 8,43 \\
\hline 4.- Ensanche & 9,77 & 9,34 & $-2,21$ \\
\hline 5.- San José & 11,22 & 9,63 & $-12,15$ \\
\hline 6.- Las Fuentes & 8,38 & 8,28 & 1,22 \\
\hline 7.- Almozara & 2,49 & 2,92 & 20,09 \\
\hline 8.- Oliver - Valdefierro & 2,42 & 2,36 & 0,00 \\
\hline 9.- Torrero - La Paz & 5,13 & 4,61 & $-7,96$ \\
\hline 10.- Margen Izquierda & 8,37 & 10,33 & 26,46 \\
\hline TOTAL & 100 & 100 & 1,22 \\
\hline
\end{tabular}

Fuente: Báguena (1998)

\section{Delimitación de las áreas de influencia de las grandes superficies de Zaragoza: aplicación sencilla al modelo de Huff}

Como se indicaba en el preámbulo metodológico, la variación de la influencia de un centro comercial en el espacio y el alcance o extensión de la misma, pueden deducirse - en un principio - a partir de las probabilidades teóricas obtenidas aplicando el modelo de Huff, con su conocida formulación (véase el pie de la tabla 3). Señalemos únicamente que en nuestro caso la variable $\left(S_{\text {. }}\right.$ ), o superficie bruta alquilable, se ha elegido por su facilidad de obtención y por capturar bien la cantidad y variedad de servicios del centro comercial. En cuanto a las distancias en tiempo $\left(\mathrm{T}_{\mathrm{ij}}\right)$ han sido estimadas imaginando desplazamientos desde cada centroide a los centros comerciales en vehículo particular, con consideración de la hora y de las rutas por las 
que tal desplazamiento se efectúa. Y, por último —dado el carácter exploratorio del trabajo-, se ha considerado conveniente asignar a la constante $k$ un valor igual a 1 . Digamos al respecto que si bien teóricamente se acepta que tal parámetro pueda tomar valores de 1 a 10, según la incidencia de la distancia en el desplazamiento o - mejor - en la interacción espacial entre el origen y el destino, es sabido que su efecto depresor en la frecuentación de centros comerciales en áreas urbanas y metropolitanas no es homogéno ( $\mathrm{y}$ de hecho se verá en la segunda parte de este estudio cómo hay distritos alejados con una proporción de visitantes mayor que otros más cercanos). De ahí la resolución tomada y las probabilidades de frecuentación obtenidas, tal y como se aprecian en la tabla 3 referida al centro comercial Grancasa.

Tabla 3. Cálculo de la probabilidad de que los residentes en Zaragoza acudan al centro comercial Grancasa

\begin{tabular}{|c|c|c|c|c|}
\hline DISTRITO ORIGEN & $\begin{array}{l}\text { DISTANCIA AL } \\
\text { CENTRO COMERCIAL } \\
\text { GRANCASA } \\
\text { (EN MINUTOS) } \\
\text { (a) }\end{array}$ & $\begin{array}{c}\text { ATRACTIVO DEL } \\
\text { CENTRO COMERCIAL } \\
\text { GRANCASA } \\
\left(S_{1} / T_{1]}\right) \\
\text { (b) }=(78188 /(a))\end{array}$ & $\begin{array}{c}\text { SUMA DE LOS } \\
\text { ATRACTIVOS DE LOS } \\
\text { CENTROS } \\
\text { COMPETIDORES } \\
\begin{array}{c}\text { (c) }=E\left(S_{j} / T_{b j}\right) \\
(j=1,2,3)\end{array}\end{array}$ & $\begin{array}{l}\text { PROBABILIDAD DE QUE LOS } \\
\text { RESIDENTES DE CADA } \\
\text { DISTRITO ACUDAN AL } \\
\text { CENTRO COMERCIAL } \\
\text { GRANCASA } \\
\text { (b) } / \text { (c) }\end{array}$ \\
\hline 1.- Casco Histórico & 10 & 7818,80 & 12267,67 & 0,637 \\
\hline 2.- Centro & 20 & 3909,40 & 8358,27 & 0,468 \\
\hline 3.- Delicias & 15 & 5212,53 & 12742,76 & 0.409 \\
\hline 4.- Ensanche & 25 & 3127,52 & 8046,83 & 0,389 \\
\hline 5.- San José & 25 & 3127,52 & 6431,73 & 0,486 \\
\hline 6.- Las Fuentes & 20 & 3909,40 & 6826,57 & 0,573 \\
\hline 7.- Almozara & 7 & 11169,71 & 20886,03 & 0,535 \\
\hline 8.- Oliver - Valdefierro & 25 & 3127,52 & 14615,27 & 0,214 \\
\hline 9.- Torrero - La Paz & 35 & 2233,94 & 5115,70 & 0.437 \\
\hline 10.- Margen Izquierda & 4 & 19547,00 & 23733,62 & 0,824 \\
\hline 11.- Barrios Rurales NW & 5 & 15637,60 & 20602,19 & 0,759 \\
\hline 12.- Barrios Rurales NE & 20 & 3909,40 & 7458,83 & 0.524 \\
\hline 13.- Barrios Rurales SW & 18 & 4343,78 & 9918,63 & 0,438 \\
\hline 14.- Barrios Rurales SE & 40 & 1954,70 & 3676,70 & 0,532 \\
\hline 15.- Utebo & 20 & 3909,40 & 11544,87 & 0,339 \\
\hline
\end{tabular}

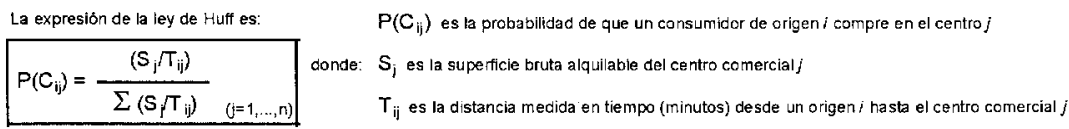

(Se asume que el valor de la constante $k$ que calibra el tiempo a las candiciones del lugar es 1, por lo que se omite en la formula) CENTROS COMPETIDORES: GRANCASA, AUGUSTA Y ALCAMPO 
Los valores resultantes al término de los cálculos representan la probabilidad de que los compradores de cada distrito acudan al citado centro comercial. Se han omitido otras dos tablas semejantes con los cálculos de las probabilidades de frecuentación de los dos centros con los que Grancasa compite, a saber Alcampo y Augusta. Pero conviene no olvidar que para un mismo origen las probabilidades de frecuentación a los tres centros suman uno, ya que se asume que las compras se realizan únicamente en tales centros y no en ningún otro lugar.

Las cuantías de las probabilidades obtenidas para el centro Grancasa oscilan entre un valor máximo de 0,824 en el distrito de Margen Izquierda y un mínimo de 0,214 en Oliver-Valdefierro. Pero en general, y como se ve en la tabla, son bastante altas, circunstancia que refleja una buena apreciación de sus cualidades -masa", proximidad, acceso- respecto de las de los otros dos centros competidores. No obstante, que los resultados obtenidos confieran al centro Grancasa una buena posición competitiva en el espacio urbano de Zaragoza era algo relativamente esperable, dada su mayor "masa" - representada por su superficie comercial-, su situación en el casco urbano -que se traduce en tiempos de acceso relativamente bajos-, y su buena accesibilidad incluso para distritos alejados, por lo que la fricción de la distancia le afecta poco. Todo ello se observa aún mejor en la figura 2, en la que los valores de probabilidad, convenientemente asignados a los centroides correspondientes, han sustentado el dibujo de líneas de isoprobabilidad -que unen puntos cuya probabilidad teórica de acudir al centro estudiado es la misma- Nótese que tal mapa, al igual que los otros realizados y que aquí no se presentan, no sería el único posible con tales datos ya que todos los mapas tienen múltiples soluciones, pero se ha elegido éste por considerarlo adecuado. En su trazado se ha evitado cerrar las curvas en algunos casos por la ausencia de datos para ciertas zonas del mapa, e igualmente se ha reflejado el efecto barrera que representa el río Ebro y la discontinuidad que imprime a los flujos de toda índole que se dan entre las dos orillas.

Las líneas de isoprobabilidad teórica rodean claramente el centro comercial y presentan un apreciable gradiente desde la de valor más alto que, como se ve, encierra a buena parte del distrito de la Margen Izquierda donde se sitúa el centro estudiado. Se observa cómo también desde distritos más distantes, como son los Barrios Rurales del SW o Utebo, es relativamente fácil acceder al centro Grancasa mediante vías de circulación rápida que mejoran axialmente las posibilidades de interacción y la accesibilidad espacial, por lo que los tiempos de recorrido son bajos y repercuten positivamente sobre los valores de probabilidad obtenidos. Esto último viene a confirmar algo que también se comprobó en los mapas correspondientes a los otros dos centros estudiados, y es que la variación espacial de la influencia que reflejan los valores de la probabilidad teórica debe ser interpretada no sólo en relación con la distancia efectiva a la que se encuentra el centro comercial, sino también según la impedancia espacial o dificultad de acceso desde cada centroide (aunque limitada en este caso a una función del tiempo del desplazamiento). 


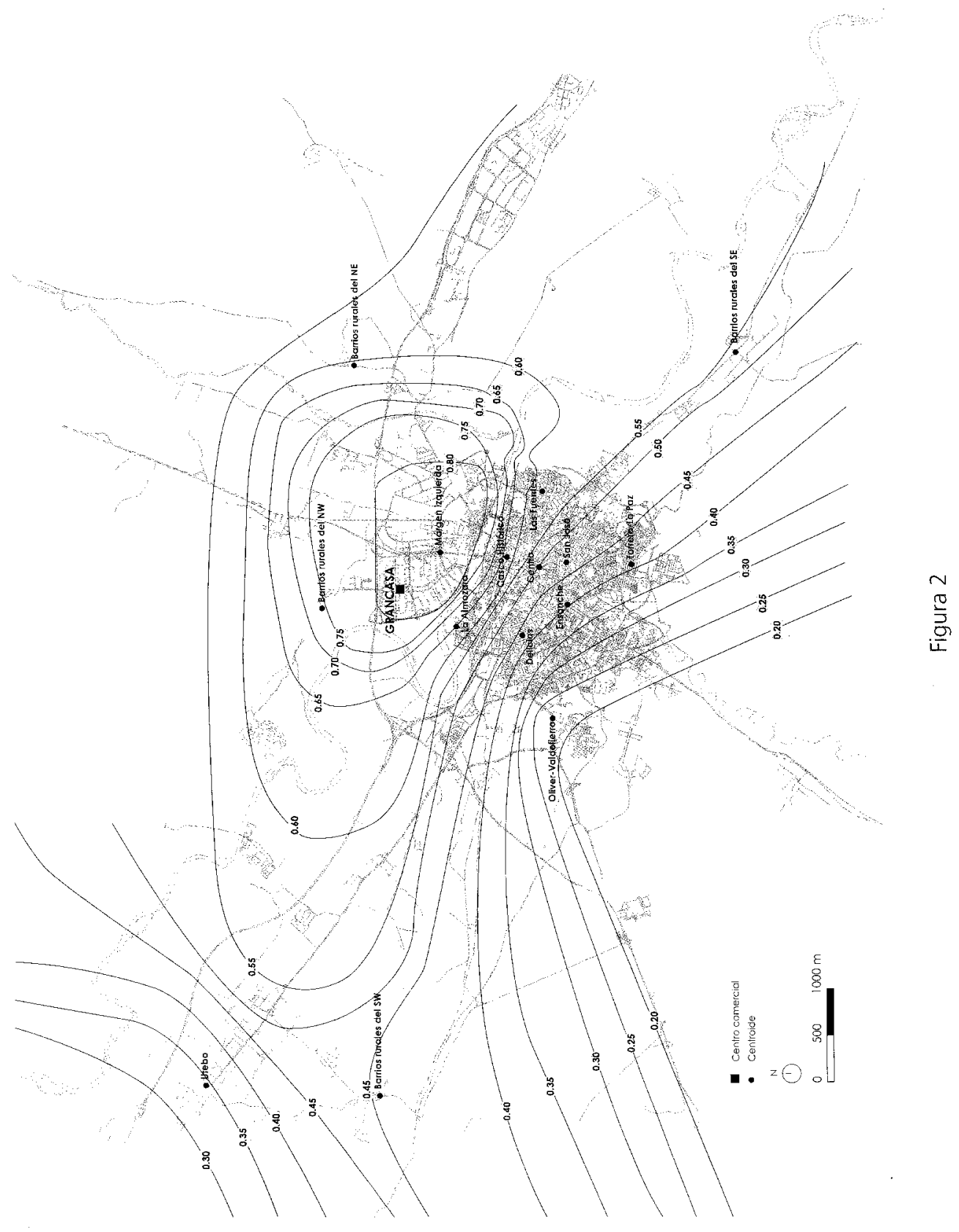




\section{Delimitación de las áreas de influencia de las grandes superficies comerciales: análisis a partir de cuotas de mercado}

Esta segunda parte del estudio de las áreas de influencia de las tres grandes superficies, aporta una mayor aproximación a la realidad por dos motivos. Primero, porque el marco interpretativo es menos restrictivo, ya que se asume la posible influencia en los desplazamientos de la oferta comercial local, así como una acepción de impedancia espacial más amplia (vid. supra las consideraciones de carácter conceptual y metodológico). En segundo lugar, también el realismo es mayor porque al análisis se incorporan los resultados de una encuesta, así como diversos datos sobre rentas y consumo de los residentes en todos los distritos urbanos. En esto hemos seguido de cerca a Berry y Pahr (1988) con el fin de obtener la "cuota de mercado" o proporción de gasto derivada por el centro comercial de cada distrito, la cual debidamente cartografiada proporciona otra representación del área de influencia comercial que matiza alguna de las conclusiones obtenidas con el modelo teórico. Los puntos de partida (distritos, centroides), se mantienen así como los mismos tres centros comerciales de destino y la restricción de que todos los compradores en los centros proceden de dichos distritos.

\subsection{Estimación de la capacidad de gasto teórica anual por distrito}

Para comenzar el análisis era necesario disponer de información sobre la capacidad de gasto de los distintos distritos urbanos de Zaragoza. Para ello se consultaron diversas fuentes. La "Encuesta Continua de Presupuestos Familiares" Base 1997 (ECPF). del Instituto Nacional de Estadística (INE) proporciona una tabla de gasto anual medio por hogar y por persona (miles de pesetas) según nivel de estudios terminados del sustentador principal en el conjunto de España. Los datos resultaron ser asimilables a los que se dan en Aragón, ya que el gasto medio por persona reseñado para Aragón es sólo algo mayor que el promedio (índice 106,3).

Estos datos se combinaron, tras unos imprescindibles ajustes, con los relativos a población de 10 o más años por estudios terminados según distrito de residencia que proporciona el Padrón Municipal de habitantes 1996 del Ayuntamiento de Zaragoza. En el caso de los barrios rurales, donde la fuente no detalla el nivel de estudios de los residentes, se optó por asignar a los de más de 10 años el gasto promedio de España, que según los datos del INE es de 898,3 miles de pesetas.

Señalemos por último que, dada la naturaleza y diversidad de los bienes ofertados en los centros comerciales estudiados, se estimó conveniente extraer del gasto teórico total la parte destinada a bienes de consumo, que es el 29,24\% según la Encuesta Continua de Presupuestos Familiares Base 1997 (ECPF). Se llega así al final del proceso recogido en la tabla 4 . 
Tabla 4

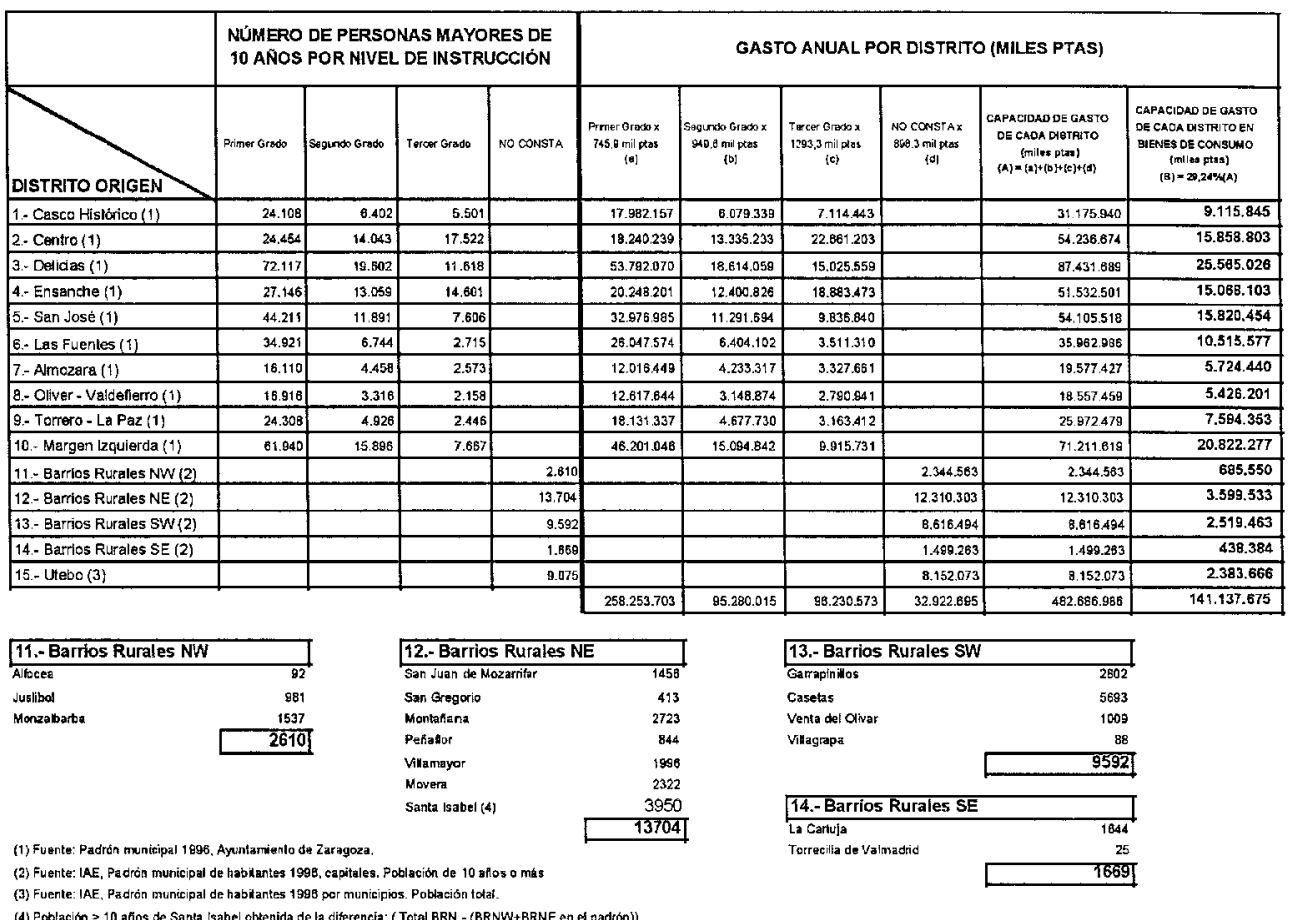

\subsection{Encuesta sobre el origen de los compradores en el centro comercial Grancasa y sus resultados}

Con objeto de disponer de información sobre la procedencia de los compradores, se llevó a cabo una pequeña encuesta a la salida de cada centro comercial el sábado 27 de noviembre de 1999, entre las 11 y las 13 horas aproximadamente, según el método de muestreo sistemático y con intervalos de tiempo, que en este caso fueron de tres minutos aproximadamente. La encuesta se limitó a indagar el distrito de procedencia de los encuestados, con objeto de conocer la presencia de compradores de los distintos distritos en los tres centros comerciales y de sustentar así la estimación de sus expectativas de gasto en cada centro comercial; expectativas - como se veráproporcionales a la presencia relativa de sus compradores. Al término de esas dos horas los alumnos encuestadores habían obtenido un total de 386 respuestas. No obstante, los resultados de la encuesta y, en concreto, su representatividad respecto de la presencia efectiva en cada centro de compradores de los distintos distritos, deben 
contemplarse con cautela, ya que los recursos disponibles no eran los necesarios para acometer un muestreo de esas características. Pero hemos tenido en cuenta las imperfecciones del sistema a la hora de valorar los resultados de todo el estudio. Veremos, por ejemplo, cómo los valores de 0 reseñados en algún distrito - por no haberse recogido respuestas de sus vecinos o potenciales compradores en la muestra-, se interpretan en términos relativos, o como indicadores de una cuota de mercado "menor", aunque no nula.

\subsection{Estimación aproximada de la cuantía de las ventas de los tres centros comerciales}

Como la cuota de mercado pretende reflejar la proporción de las ventas totales que cabe esperar de los residentes en un distrito dado, resulta imprescindible disponer de una estimación de las ventas totales que realizan de los centros comerciales estudiados. Es habitual, y así lo hemos hecho aquí, realizar dicha estimación de manera indirecta a partir de las informaciones proporcionadas en los centros sobre el número de visitantes o de compradores anuales. Además se ha interpretado que sólo uno de cada cuatro visitantes realiza realmente compras en el establecimiento, y por otra parte se ha asumido que cada comprador efectúa un gasto promedio de 4.000 ptas. Ciertamente, y según las circunstancias o la información disponible, pueden variarse tales criterios, obteniéndose así cuantías de ventas distintas a las que aquí se presentan. No obstante las consultas que se han realizado avalan las determinaciones aquí tomadas.

\subsection{Obtención de las cuotas de mercado, cartografía y comentarios}

La tabla 5 reproduce la secuencia del cálculo, donde se puede ver que la proporción de las ventas esperables de cada distrito en función de la presencia relativa de sus većinos entre los clientes encuestados, es a su vez dividida por la cuantía de los gastos teóricos en bienes de consumo de dicho distrito. Se obtiene así para cada centroide un nuevo indicador, o cuota de mercado del centro comercial $j$ en el distrito $i$, que indica qué proporción del gasto en bienes de consumo de $i$ se realiza en $j$.

Tales cuotas de mercado se llevan al mapa base con el que se ha trabajado anteriormente y se procede al trazado de isolíneas que serán indicativas, en esta ocasión, de la penetración de los centros comerciales respectivos. Incorporamos nuevamente el que corresponde al centro Grancasa (figura 3).

Para interpretar los resultados obtenidos debe recordarse que un valor alto de la cuota de mercado reflejaría una alta penetración del centro comercial en ese distrito; 
Tabla 5. Obtención de la cuota de mercado correspondiente al cenro comercial Grancasa considerando capacidad de gasto de cada distrito en bienes de consumo

\begin{tabular}{|c|c|c|c|c|c|}
\hline DISTRITO ORLEN & $\begin{array}{l}\text { CAPACIDAD DE GASTO } \\
\text { TOTAL DE CADA } \\
\text { DISTRITO (miles ptas) } \\
\text { [A) }\end{array}$ & $\begin{array}{c}\text { CAPACIDAD DE GASTO } \\
\text { DE CADA DISTRITO EN } \\
\text { BIENES DE CONSUMO } \\
\text { (miles ptas) } \\
\text { (B) }=29.24 \%(\text { A) }\end{array}$ & $\begin{array}{l}\text { PROCEDENCIA DE LOS } \\
\text { COMPRADORES DEL } \\
\text { CENTRO COMERCIAL } \\
\text { GRANCASA (\%) } \\
\text { (a) }\end{array}$ & $\begin{array}{l}\text { CUANTIA ESTIMADA DE } \\
\text { LAS COMPRAS DE CADA } \\
\text { DISTRITO EN EL } \\
\text { CENTRO COMERCIAL } \\
\text { GRANCASA (miles ptas) } \\
\text { (C) }=(\text { a })^{*}(\mathrm{~V}) / 100\end{array}$ & $\begin{array}{l}\text { CUOTA DE MERCADO } \\
\text { DEL CENTRO } \\
\text { COMERCIAL GRANCASA } \\
\text { (miles ptas) } \\
(\mathrm{M})=(\mathrm{C}) /(\mathrm{B})\end{array}$ \\
\hline 1.- Casco Histórico & 31.175 .940 & 9.115 .845 & 3,49 & 523.256 & 0,0574 \\
\hline 2.- Centro & 54.236 .674 & 15.858 .803 & 1,16 & 174.419 & 0,0110 \\
\hline 3.-Delicias & 87.431 .689 & 25.585 .026 & 18,60 & 2.790 .698 & 0,1082 \\
\hline 4.-Ensanche & 51.532 .501 & 15.068 .103 & 1,16 & 174.419 & 0,0116 \\
\hline 5.- San José & 54.105 .518 & 15.820 .454 & 1,16 & 174.419 & 0,0110 \\
\hline 6. Las Fuentes & 35.962 .986 & 10.515 .577 & 2.33 & 348.837 & 0,0332 \\
\hline 7.-Aimozara & 19.577 .427 & 5.724 .440 & 9,30 & 1.395 .349 & 0,2438 \\
\hline 8. - Oliver - Valdefierro & 18.557 .459 & 5.426 .201 & 0,00 & 0 & 0,0000 \\
\hline 9.- Torrero - La Paz & 25.972 .479 & 7.594 .353 & 8,14 & 1.220 .930 & 0,1608 \\
\hline 10.- Margen Izquierda & 71.211 .619 & 20.822 .277 & 54,65 & 8.197 .674 & 0,3937 \\
\hline 11.- Barrios Rurales NW & 2.344 .563 & 685.550 & 0,00 & 0 & 0,0000 \\
\hline 12.- Barrios Rurales NE & 12.310 .303 & 3.598 .533 & 0,00 & 0 & 0,0000 \\
\hline 13.- Bartios Rufales SW & 8.616 .494 & 2.519 .463 & 0,00 & 0 & 0,0000 \\
\hline 14.- Barrios Rurales SE & 1.499 .263 & 438.384 & 0,00 & 0 & 0,8000 \\
\hline 15.- Utebo & B. 152.073 & 2.383 .666 & 0,00 & 0 & 0,0000 \\
\hline TOTAL & 482.686 .986 & 141.137 .675 & 100,00 & 15.000 .000 & \\
\hline
\end{tabular}

(B) es al gasto en bienes de consumo: alimentos, hebidas, articulos de vestir y calcado

Ventas estimadas del Centro Comercial Augusta, (V): 15.000 .000 miles ptas

(ESTIMACION: $1 / 4 \times 15.000 .000$ visitantes $\times 4.000$ ptan ganto medio\})

y a la inversa, un valor bajo sería propio de una baja penetración comercial. En términos de competitividad las cuotas serían indicadores de la posición competitiva del centro comercial en un área dada, de modo que altas cuotas indicarían una posición competitiva ventajosa, mientras que bajas cuotas sugerirían la existencia para ese distrito de otras alternativas más atractivas, que - dados los supuestos expuestos en el capítulo metodológico- podrían provenir de las otras dos grandes superficies comerciales en competencia, o bien de la respectiva oferta local.

Examinando ya los valores obtenidos y que se reflejan en la tabla y en el mapa que de ella se deriva, es notorio el rango entre valores extremos. Conviene advertir que las cuotas de valor $0(0,000)$, correspondiente a los cuatro distritos de barrios rurales, Oliver-Valdefierro así como al municipio de Utebo, resultan de no haber ninguno de sus vecinos entre los encuestados del día 27 de noviembre. Dadas las circunstancias que afectaron a la propia encuesta y para no incurrir en una subestimación de la atracción del centro comercial Grancasa sobre tales distritos, puede interpretarse el 0 en términos relativos, como expresión de una "menor" cuota de mercado en tales distritos. En cuanto a los valores más altos se alcanzan para los distritos de Margen Izquierda $(0,3937)$ y La Almozara $(0,2438)$, circunstancia esperable por su proximidad a dicha gran superficie. Sin embargo los mínimos relativos obtenidos 


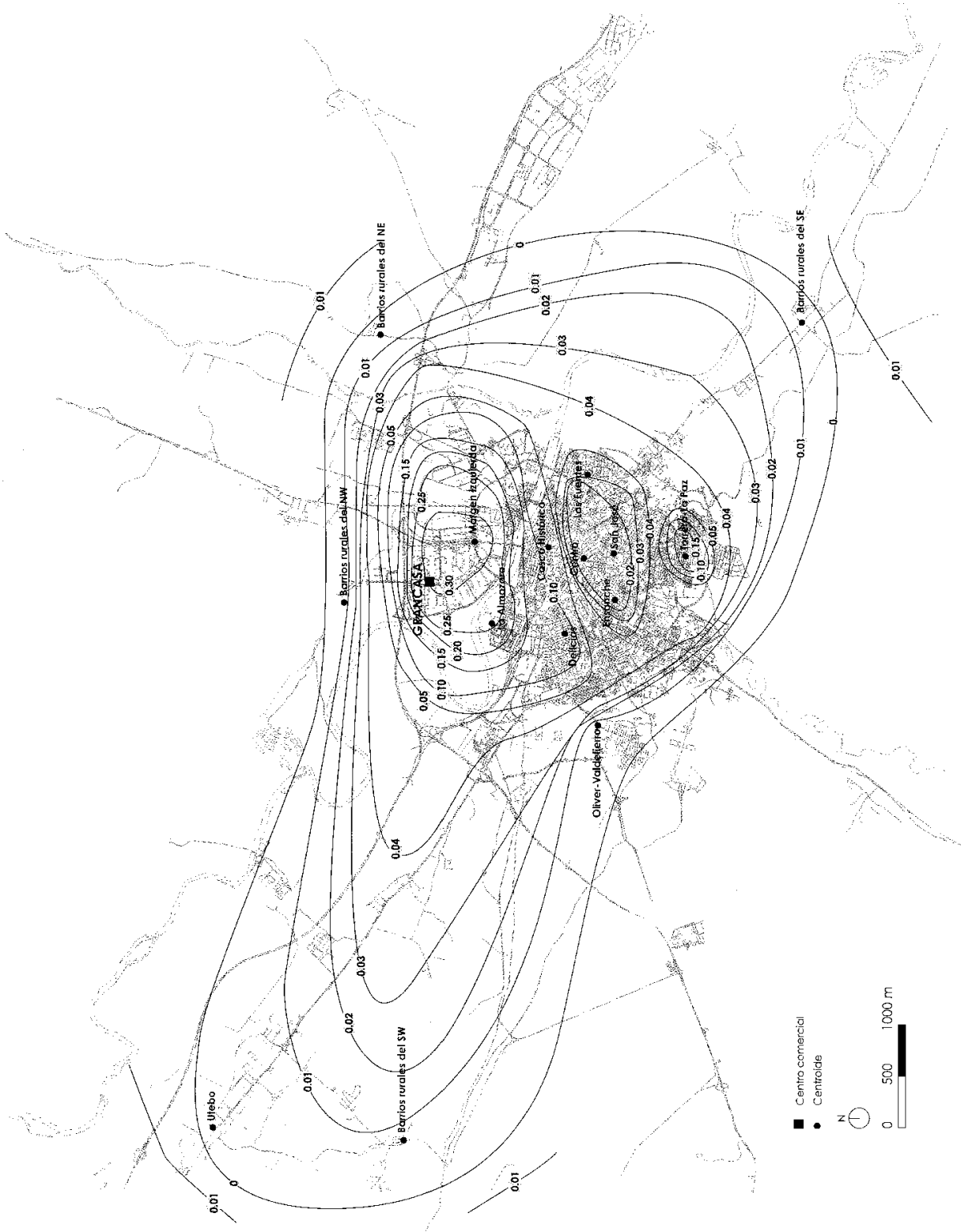


en algunos distritos del centro (0,0110 en Centro y San José, 0,0116 en Ensanche), así como los valores altos de otros más periféricos $(0,1608$ en Torrero-La Paz), sugieren la influencia variable de factores como la oferta local y la impedancia espacial del acceso, aunque en términos más amplios que los antes señalados, ya que aquí la impedancia sería no sólo una función del tiempo del desplazamiento, sino también de su coste, conveniencia, fiabilidad, seguridad, etc. Así las cosas, en el caso de los distritos centrales puede argumentarse que la baja cuota de mercado obtenida por el centro comercial Grancasa se debe a que la oferta local pesa más que la escasa fricción que opone la distancia; y a la inversa, en el caso de Torrero-La Paz, la cuota relativamente más alta de este centro comercial se debería a que a la mayor fricción de la distancia existente no se le opone, porque no la hay, una oferta comercial local de similares características. En términos de impedancia espacial podríamos sugerir también que los atributos del acceso habitual desde este distrito al centro comercial Grancasa - en especial los atributos distintos del tiempo de recorrido-, confieren a este centro la mayor cuota de mercado en el barrio (ya que de los tres centros comerciales en litigio, todos bastante alejados, es Grancasa el que alcanza la mayor cuota de mercado en el distrito con bastante diferencia).

En vista de las notables variaciones en las cuantías de las cuotas de mercado, la realización del mapa ha seguido los siguientes criterios: isolíneas que van de 0,01 en 0,01 desde la de cero a la de 0,05; y a partir de ésta, de 0,05 con incrementos de 0,05 hasta el valor máximo, que es la de 0,3 . Por otra parte, dado que seis distritos presentan valores nulos, era necesario trazar la línea de cero y se ha optado por hacerlo de forma envolvente. También se han indicado líneas de 0,01 hacia fuera, tratando de señalar con ello que hay más mundo que el espacio estudiado y que probablemente los valores de cuotas de mercado sean mayores que cero en algún punto de ese espacio. Se ha obtenido así una imagen muy interesante de cómo varía la ya descrita influencia comercial del centro Grancasa por el espacio urbano de Zaragoza. Se aprecia con claridad el contraste claro entre las cuotas obtenidas en las proximidades del centro comercial y las de las áreas más periféricas, si bien ese descenso con la distancia no es uniforme, sino que queda alterado especialmente hacia el sur, por la propia configuración urbana de la ciudad. Así la isolínea de valor 0,04 , que rodea grosso modo el espacio construido, encierra dos áreas de altas cuotas relativas (la próxima al centro comercial y la ya citada y más meridional de Torrero-la Paz), entre las que se encaja otra claramente diferenciada de bajas cuotas correspondiente a los distritos del centro (Centro, Ensache, San José y Las Fuentes).

\section{Conclusiones: valoración de lor resultados cartográficos, de la metodología utilizada y perspecivas}

Si se comparan los mapas que representan el área de influencia, o más generalmente, la posición competitiva de las grandes superficies comerciales en el espacio 
urbano de Zaragoza, se comprueba, tanto en los mapas mostrados en este trabajo como en los demás realizados, que los valores obtenidos para la cuota de mercado tienen un rango mucho mayor que los correspondientes a la probabilidad teórica, lo que confirma que realmente los consumidores eligen acudir o no a un determinado centro por razones diferentes al simple hecho de la distancia en tiempo que les separa de dichos centros y del tamaño de los mismos. Además, en el mapa de probabilidades teóricas no hay valores cero, ya que se asume que de la "masa" de cualquiera de los centros emanará siempre un tanto de atracción e, igualmente, que los consumidores no podrán elegir una oferta distinta a la de los tres centros en competencia. Pero en el análisis según cuotas de mercado se relajan implícitamente estos supuestos, y los resultados de la encuesta apuntan a que en la realidad la población tiene una oferta comercial más o menos amplia en su propio barrio o en otros distritos a los que se puede desplazar más fácilmente, de lo que resulta que la penetración comercial de algunas grandes superficies en tales distritos pueda dar cuotas de valor cero o muy próximas a cero.

En cuanto a la forma de las isolíneas, también presentan configuraciones distintas en los dos casos. En el mapa según probabilidades tienen un gradiente mucho menor, es decir, los máximos y los mínimos son menos acusados. Si se hiciera un símil topográfico los mapas de probabilidad teórica serían el reflejo de un paisaje alomado siempre con altitudes superiores al nivel del mar, mientras que los obtenidos con la cuota de mercado serían reflejo de un paisaje montañoso muy abrupto que partiendo del nivel del mar tuviera alturas enormes frente a depresiones y gargantas muy profundas.

Sin embargo, si se estudian con algo más de detalle presentan algunas similitudes, la más importante de las cuales es que, una vez asumida la diferencia en los valores de partida, los mapas mantienen una estructura de un máximo alrededor o cerca del centro comercial más o menos profundo, lo que responde a la idea de que la fricción de la distancia es menor en lugares más cercanos. En la misma línea, otra similitud que se puede encontrar es que los valores mínimos están alejados del centro comercial objeto de estudio aunque hay que destacar las excepciones que se han comentado en cada caso. En otro orden de cosas la comparación de mapas correspondientes a superficies comerciales distintas, evidencia cómo se reparten el mercado urbano y en qué áreas cabe, o no, inducir una mayor penetración, y ello mediante las estrategias que se consideren oportunas.

En todo caso los resultados cartográficos reflejan fielmente las opciones metodológicas. A este respecto creemos haber demostrado el atractivo de esta propuesta de cara a estimar la penetración comercial de una gran superficie en el espacio urbano, como se ha hecho aquí con el caso de Zaragoza. No obstante cabe señalar algunas cuestiones que convendría tener en cuenta para estudios posteriores y contando con medios adecuados:

a) La necesidad de completar la encuesta con toda la complejidad de los muestreos probabilísticos, para poder plantear objetivos más ambiciosos con pretensiones inferenciales y no exploratorias como en este caso. 
b) La conveniencia de ajustar adecuadamente la cuantía de las ventas de los centros comerciales, procurando encajar con la escala del análisis. A este respecto es esencial la contribución de las propias empresas, no para revelar una información considerada como confidencial, sino para facilitar criterios que permitan un mayor acierto en la estimación.

c) La urgencia en superar problemas en cuanto a las fuentes, que como ocurre frecuentemente, impiden sacar partido a niveles altos de desagregación -como los que brindan los padrones municipales de habitantes-, al ser imposible o dificultoso su ajuste con las informaciones de otras procedencias. En este ámbito debería ser innecesario proceder por aproximación e incurrir en los errores que ello representa.

d) La posibilidad de manejar información demográfica a niveles más desagregados, con lo que se dispondría de un mayor número de puntos de control.

e) Por último resulta obvio que la precisión en la identificación del centroide asî como en la consideración de la impedancia espacial o, en general, de las circunstancia que influyen en la facilidad o dificultad de acceso al centro comercial, no harán más que redundar en la validez de los resultados obtenidos.

En definitiva no nos cabe duda de que con una adecuada información quedarían mejor realzadas las posibilidades analíticas del método propuesto, así como su potencial para contribuir a una mejor apreciación de la posición competitiva de las grandes superficies comerciales en nuestros espacios urbanos, como hemos hecho en el caso de Zaragoza.

\section{Notas}

1 El trabajo de campo requerido para la investigación fue realizado por un equipo de alumnos de segundo ciclo, dirigidos por la Dra. Escalona y compuesto por: B. Aranda, L. Borroy, P. Brun, L. Castellano, S. Delgado, C. Díez, A. Escudero, H. Esteban, A. López, S. López, S. Obón, P. Parra, C. Pérez, R. Pons, M. Ziegller y M. Baumann.

2 Como recuerdan ambas autoras la citada Asociación define el centro comercial como un conjunto de establecimientos comerciales independientes, planificados y desarrollados por una o varias entidades con criterio de unidad, cuyo tamaño, mezcla comercial, servicios comunes y actividades complementarias están relacionadas con su entorno, y que dispone permanentemente de una imagen y gestión unitaria (Flavián y Polo, op. cit., 89)

\section{Bibliografía}

Antón Burgos, F.J., coord. (1999): La geogra-

fia de los servicios en España. Madrid,
Universidad Complutense de Madrid / Asociación de Geógrafos Españoles. 
Berry, B y Parr, J. (1988): Market Centers and retail location. New Jersey, Prentice Hall.

Báguena Sánchez, J.A. (1998): La estructura del comercio minorista y el urbanismo commercial. Economia Aragonesa, 2, p. 105-124.

Bielza de Ory, V. y Escolano Utrilla, S. (1985): Espacio periurbano y comercio: El caso de Zaragoza. IX Coloquio de Geografía, Ponencias. Valencia, Centro Editorial de Servicios y Publicaciones Universitarias, SA.

Delgado Urrecho, J.M., (1991): Incidencia de las grandes superficies en el potencial de ventas del comercio minorista: análisis cuantitativo. En VV.AA., Primeras jornadas de Geografia y Comercio: grandes superficies comerciales. Madrid, Universidad Complutense, p. 287-303.

Escolano Utrilla, S. (1995): Un sistema de información geográfica para el comercio y los servicios de Zaragoza (SIGCAM). XIV Congreso Nacional de Geografia: Cambios regionales a finales del siglo $X X$. Salamanca, Universidad de Salamanca/Asociación de Geógrafos Españoles, p. 470-474.

Escolano Utrilla, S. (1999): Evolución de los estudios geográficos sobre el comercio en España. En Antón Burgos, F.J., coord., La geografia de los servicios en España. Madrid, Universidad Complutense de Madrid / Asociación de Geógrafos Españoles, p. 431-454́.

Flavián Blanco, C, y Polo Redondo, Y. (1998), Nuevas fórmulas comerciales para un nuevo consumidor: el caso de los centros comerciales. Economia Aragonesa, 2, 77-104.

Instituto Aragonés de Estadística (1999): Padrón Municipal de Habitantes 1996. Aragón: capitales. Zaragoza, Diputación General de Aragón, Instituto Aragonés de Estadística.

Instituto Nacional de Estadística (1999): Encuesta Continua sobre Presupuestos Familiares. Base 1997 (ECPF). Madrid, Instituto Nacional de Estadística.

Moreno Jiménez, A. (1995): Planificación y gestión de servicios a la población desde la perspectiva territorial: Algunas propuestas metodológicas. Boletín de la Asociación de Geógrafos Españoles, 20, 115-134.

Moreno Jiménez, A. y González Briega, J.C. (1999): "La competencia espacial de grandes superficies comerciales en áreas metropolitanas: un estudio de cason. En Antón Burgos, F.J., coord., La geografia de los servicios en España. Madrid, Universidad Complutense de Madrid / Asociación de Geógrafos Españoles, p. 569-576.

Serrano Martínez, J,M, y Martín Hernando, M.A., (1991): Grandes superficies (hipermercados) y configuración de nuevas áreas de mercado: El caso de Murcia. En V.AA., Primeras jornadas de Geografia y Comercio: grandes superficies comerciales. Madrid, Universidad Complutense, pp. 37-60.

VV.AA. (1991), Primeras jornadas de Geografia y Comercio: grandes superficies comerciales. Madrid, Universidad Complutense. 